\title{
Atypical umbilical naevi: histopathological analysis of 20 cases*
}

\author{
David P Arps, ${ }^{1}$ Douglas R Fullen ${ }^{1,2}$ \& May P Chan ${ }^{1,2}$ \\ Departments of ${ }^{1}$ Pathology, and ${ }^{2}$ Pathology Dermatology, University of Michigan, Ann Arbor, MI, USA
}

Date of submission 17 March 2014

Accepted for publication 10 July 2014

Published online Article Accepted 11 August 2014

Arps D P, Fullen D R, Chan M P

(2015) Histopathology 66: 363-369. DOI: 10.1111/his.12503

\section{Atypical umbilical naevi: histopathological analysis of 20 cases}

Aims: Melanocytic naevi on the umbilicus have been described as a form of flexural naevi, with the most common feature being a 'nested and dyshesive pattern'. We have encountered a distinct group of umbilical naevi with more significant atypia and prominent fibrosis, not reported previously. This study aimed to characterize these naevi more clearly.

Methods and results: Eighty-one umbilical naevi from 2000 to 2013 were reviewed retrospectively, 20 cases of which showed lamellar fibrosis and atypia and were designated as atypical umbilical naevi (AUN). Lamellar fibrosis in AUN was extensive and frequently entrapped dermal melanocytes, resulting in low-grade cytological atypia ( $74 \%$ of cases) and impaired maturation $(47 \%)$ of the dermal component.
Other common features included bridging (95\%), shoulder architecture (94\%), lentiginous growth $(85 \%)$ and high-grade junctional cytological atypia (85\%). 'Nested and dyshesive pattern' was observed in only $20 \%$ of AUN. Ki-67 immunostaining performed on 12 AUN revealed a consistently low proliferation index of $<1 \%$. All AUN in this series lacked junctional confluence, florid pagetoid spread and dermal mitoses.

Conclusion: A subset of special site naevi in the umbilical region demonstrate characteristic lamellar fibrosis, architectural disorder and cytological atypia. Familiarity with these site-related atypical features would avoid overdiagnosis of melanoma.

Keywords: flexural, lamellar fibrosis, melanoma, naevus, special site, umbilical, umbilicus

\section{Introduction}

Melanocytic naevi occurring on the genitalia, ${ }^{1-3}$ scalp, ${ }^{4,5}$ ear, ${ }^{6,7}$ breast, $^{8}$ acral $^{9,10}$ and flexural skin ${ }^{11}$ are known to demonstrate unusual architectural and cytological features overlapping with those of melanoma. For example, large and abnormally placed junctional nests, lentiginous growth, limited pagetoid spread and various degrees of cytological atypia have been reported and attributed to the specific anatomic

Address for correspondence: M P Chan, Medical Science I, M3261, 1301 Catherine Street, Ann Arbor, MI 48109, USA.

e-mail:mpchan@med.umich.edu

*Presented previously at the 103rd Annual Meeting of the United States and Canadian Academy of Pathology, San Diego, CA, USA, 1-7 March 2014. sites of these naevi. Recognition of the spectrum of site-specific characteristics is important, as it would avoid overdiagnosis of melanoma and unnecessary aggressive treatment.

The umbilicus is a unique anatomical structure formed during the obliteration of the umbilical cord. Given the small surface area of the umbilicus, it is not surprising that melanocytic lesions occurring on umbilical skin are relatively rare and the current literature pertaining to these lesions is limited. Some authors consider the umbilicus to be a flexural site (an area of curved skin). ${ }^{11}$ When viewed together with the peri-umbilical region, it may also be considered part of the anatomical milk line. ${ }^{12}$ Only one study in the current literature has examined the histological features of 13 umbilical melanocytic naevi as part of a series of flexural naevi, and described a 
'nested and dyshesive pattern' to be the most common finding in these naevi. ${ }^{11}$ Based on the unique anatomy of the umbilicus, however, it is reasonable to hypothesize that umbilical naevi may possess additional histological features which distinguish them from other flexural naevi. Indeed, we have encountered a distinct type of umbilical naevus with more significant atypia and prominent lamellar fibrosis, which has not been reported previously. This study aimed to characterize these atypical umbilical naevi (AUN) more clearly.

\section{Materials and methods}

After approval by the institutional review board (HUM00045834, 12/15/2011), the surgical pathology database at the University of Michigan was searched for the keywords 'umbilicus' and 'umbilical' between 2000 and 2013. The results were then filtered for melanocytic naevi. Cases described as periumbilical or near the umbilicus were excluded. Only primary biopsies were included in the study.

Haematoxylin \& eosin-stained sections of all umbilical naevi were reviewed. All cases were examined for shoulder architecture (junctional component extending laterally beyond the dermal component), large round junctional nests ("nested and dyshesive pattern'), bridging of junctional nests across rete ridges, lentiginous growth of single melanocytes along the dermo-epidermal junction, confluence of junctional melanocytes spanning at least three rete ridges, pagetoid spread, involvement of suprapapillary plates, involvement of adnexal epithelium, effacement of rete ridges, lamellar fibrosis (increased dermal collagen with laminated appearance and/or in horizontal array), maturation of dermal component with depth, dermal mitoses, congenital (peri-adnexal) growth pattern and lymphoid infiltrate. Cytological atypia of the junctional and the dermal components was graded as none, low-grade (mild) or high-grade (moderate or severe), based on the degree of nuclear enlargement, hyperchromasia, prominent nucleoli and pleomorphism. ${ }^{13}$ Naevi showing both lamellar fibrosis and cytological atypia were categorized as AUN.

Immunohistochemistry was performed on AUN cases where formalin-fixed, paraffin-embedded tissue blocks were available. Sections of $4-\mu \mathrm{m}$ thickness were deparaffinized and heat-induced epitope retrieval was performed using a proprietary Tris-EDTA buffer, pH 9.0 (target retrieval solution, high pH; Dako Corporation,Carpinteria, CA, USA). After blocking endogenous peroxidase activity, the slides were incubated for $30 \mathrm{~min}$ at room temperature with a rabbit monoclonal Ki-67 antibody (SP6, 1:250; Cell Marque Corporation, Rocklin, CA, USA) and subsequently detected using the Dako EnVision FLEX+ system. Ki-67 proliferation index was determined by estimating the percentage of dermal melanocytes showing positive nuclear staining.

Clinical data including the patient's age and gender, clinical impression and subsequent excision or recurrence were obtained for all AUN from the electronic medical records.

\section{Results}

A total of 81 melanocytic naevi taken from the umbilicus were identified. Upon review, 28 cases (34\%) were consistent with common naevi with or without flexural-site features ('nested and dyshesive pattern'), and 33 cases $(41 \%)$ were consistent with dysplastic naevi. The remaining 20 cases $(25 \%)$ demonstrated characteristic lamellar fibrosis and atypia and were designated as AUN. The 20 patients with AUN were mainly females (F:M = 14:6) and ranged from 10 to 53 years of age (mean, 30.5 years). Eleven cases (55\%) of AUN appeared atypical clinically; one of these cases also demonstrated halo phenomenon. There was no reported clinical history of trauma or scar in any of the AUN. Follow-up data were available in 14 of the AUN cases, for an average period of 48.9 months (range 1-146 months). Eight patients underwent excision for positive or close margins. One patient did not undergo excision despite positive margins and was followed clinically. None of these 14 cases of AUN with clinical follow-up recurred or metastasized.

The histological findings of AUN are summarized in Table 1 and illustrated in Figures 1-5. All cases were compound naevi except for one case, which was purely junctional. Unlike lamellar fibroplasia described in dysplastic naevi, ${ }^{14}$ lamellar fibrosis in AUN was more confluent and extended more deeply into the dermis, frequently entrapping dermal melanocytes. The entrapped dermal melanocytes typically showed low-grade cytological atypia, but high-grade atypia was also observed in two cases $(11 \%)$. Nine cases $(47 \%)$ demonstrated impaired maturation and abnormal (irregular or horizontal) configuration of the entrapped dermal nests, while melanocytes outside the fibrotic zones invariably matured with depth. In addition to lamellar fibrosis, other common histological features observed in AUN include bridging, shoulder architecture, lentiginous growth, high-grade 
junctional cytological atypia and low-grade dermal cytological atypia. Large round junctional nests ('nested and dyshesive pattern') were seen in only four cases $(20 \%)$. Pagetoid spread was observed in three cases $(15 \%)$ as a focal and inconspicuous finding limited to the centre of the lesions.

Table 1. Histological features examined in atypical umbilical naevi (AUN)

\begin{tabular}{ll}
\hline Histological feature & Number of cases (\%) \\
\hline Shoulder architecture & 17 of $18^{*}(94)$ \\
\hline Large round junctional nests & $4(20)$ \\
\hline Bridging & $19(95)$ \\
\hline Lentiginous growth & $17(85)$ \\
\hline $\begin{array}{l}\text { Confluence } \\
\text { Pagetoid spread } \\
\text { Focal }\end{array}$ & 0 \\
\hline Florid & $3(15)$ \\
\hline $\begin{array}{l}\text { Involvement of suprapapillary } \\
\text { plates }\end{array}$ & 0 \\
\hline $\begin{array}{l}\text { Involvement of adnexal } \\
\text { epithelium }\end{array}$ & $8(20)$ \\
\hline
\end{tabular}

High-grade junctional 17 (85)

cytological atypia

\begin{tabular}{ll}
\hline Effacement of rete ridges & $1(5)$ \\
\hline Lamellar fibrosis & $20(100)$ \\
\hline $\begin{array}{l}\text { Impaired maturation within } \\
\text { areas of lamellar fibrosis }\end{array}$ & 9 of $19 *(47)$ \\
\hline
\end{tabular}

Maturation outside fibrotic

19 of $19 *(100)$

zones

\begin{tabular}{ll}
\hline Dermal mitoses & 0 \\
\hline $\begin{array}{l}\text { Low-grade dermal } \\
\text { cytological atypia }\end{array}$ & 14 of $19^{*}(74)$ \\
\hline $\begin{array}{l}\text { High-grade dermal } \\
\text { cytological atypia }\end{array}$ & 2 of $19^{*}(11)$ \\
\hline Congenital growth pattern & 2 of $19^{*}(11)$ \\
\hline Lymphocytic infiltrate & $8(40)$ \\
\hline
\end{tabular}

*Twenty cases were examined but not all features were assessable due to either partial biopsy or lack of a dermal component.
All 12 cases of AUN immunostained for Ki-67 demonstrated absent to rare staining in the dermal component, with an estimated proliferation index of either $0 \%$ (two cases) or 1\% (10 cases). An example is shown in Figure 3B.

\section{Discussion}

Similar to naevi of the genitalia, ${ }^{1-3}$ scalp, ${ }^{4,5}$ ear, ${ }^{6,7}$ breast, $^{8}$ and acral ${ }^{9,10}$ and flexural ${ }^{11}$ skin, naevi of the umbilicus may display 'special site' features distinct from ordinary naevi and at times concerning for melanoma. ${ }^{14}$ The current literature on umbilical naevi is limited to a study by Rongioletti et al. ${ }^{11}$, who examined 40 flexural naevi including 13 cases taken from the umbilicus. They found that more than half these flexural naevi displayed a 'nested and dyshesive pattern', referring to large confluent junctional nests with variation in size, shape and position as well as diminished cohesion of the melanocytes. Such a pattern is similar to that described previously in genital naevi. ${ }^{2}$ Fibroplasia was also reported in all cases with a 'nested and dyshesive pattern', although further discussion on the extent and characteristics of the fibroplasia was lacking. In our current series, besides cases with the aforementioned flexural-type features, one-fourth of the umbilical naevi demonstrated prominent lamellar fibrosis and significant architectural and cytological atypia. The majority of this latter group lacked the characteristic 'nested and dyshesive pattern' and exhibited a higher degree of atypia compared to the flexural naevi described by Rongioletti et al. We hereby conducted this comprehensive histopathological review to further characterize this group of AUN.

During the development of the umbilicus, the umbilical cord becomes obliterated and replaced by a collagenous plate which fuses with the neighbouring umbilical ring. ${ }^{15}$ The lamellar fibrosis observed in AUN may therefore be a physiological finding owing to the unique anatomy of the umbilicus. Microscopically, lamellar fibrosis has an organized or laminated appearance. The collagen fibres are slightly paler and finer compared to the normal reticular collagen in the deeper dermis, and in most cases are orientated parallel to the epidermis. Unlike the lamellar fibroplasia in dysplastic naevi that is confined to the papillary dermis, ${ }^{16,17}$ the lamellar fibrosis seen in AUN tends to be more prominent, often extending into the reticular dermis. It frequently entraps dermal nests of melanocytes, leading to a horizontal array or irregular configuration of the latter. The entrapped cells almost 

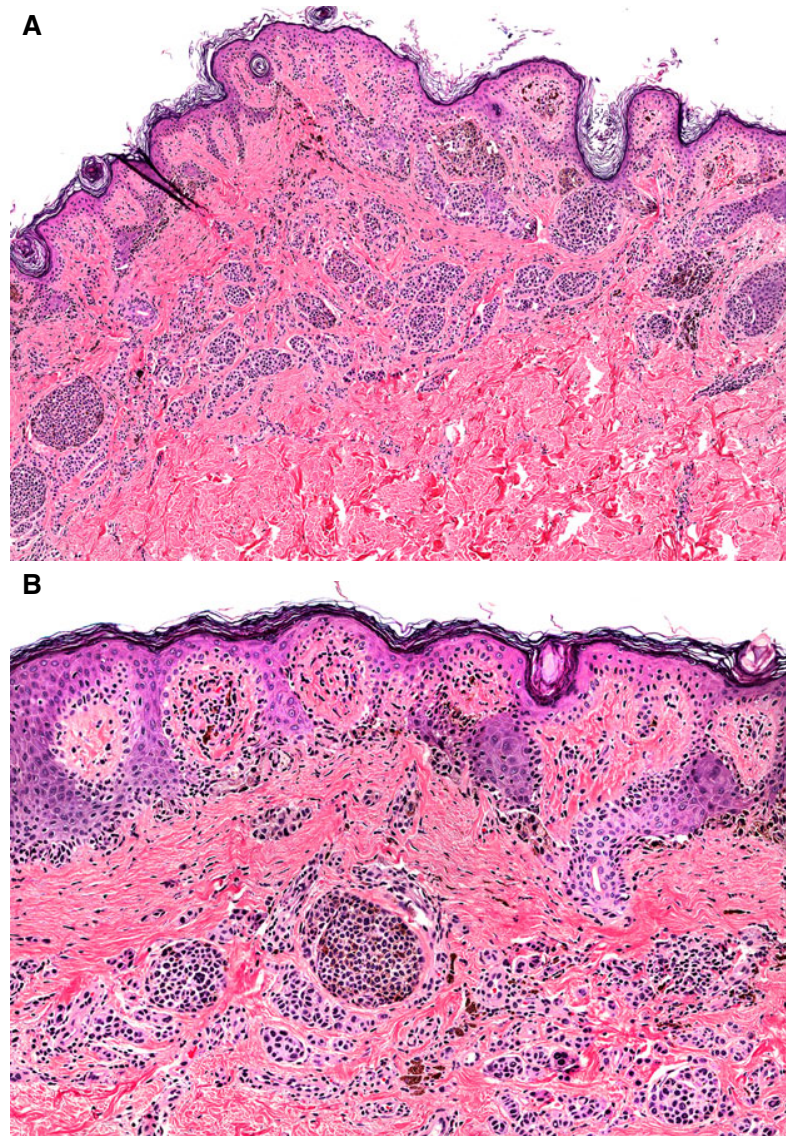

Figure 1. Atypical umibilical naevus (AUN). A, Part of this lesion shows lamellar fibrosis with entrapment of dermal nests of variable sizes. B, The lamellar fibrosis in this case is relatively subtle and demonstrates some resemblance to the lamellar fibroplasia of dysplastic naevi, except that it is more expanded and extends into the reticular dermis. The entrapped dermal nests are larger than usual and are composed of mildly atypical melanocytes, but maturation is evident at the base of the lesion. The overlying junctional component shows lentiginous growth and high-grade cytological atypia without confluence, suprapapillary plate involvement or pagetoid spread.

always appear mildly atypical. Maturation is compromised within the areas of lamellar fibrosis in nearly half the cases. However, any dermal melanocytes present outside the fibrotic zones invariably demonstrate complete maturation and banal cytology, indicating that impaired maturation and cytological atypia are solely results of entrapment by lamellar fibrosis.

With regard to the junctional component, large round junctional nests typical of flexural or genital naevi are present in only a minority of AUN. The majority of cases consist of some lentiginous melanocytes along the basal layer; however, the suprapapillary plates remain spared or are only focally involved. Junctional confluent growth is absent in all
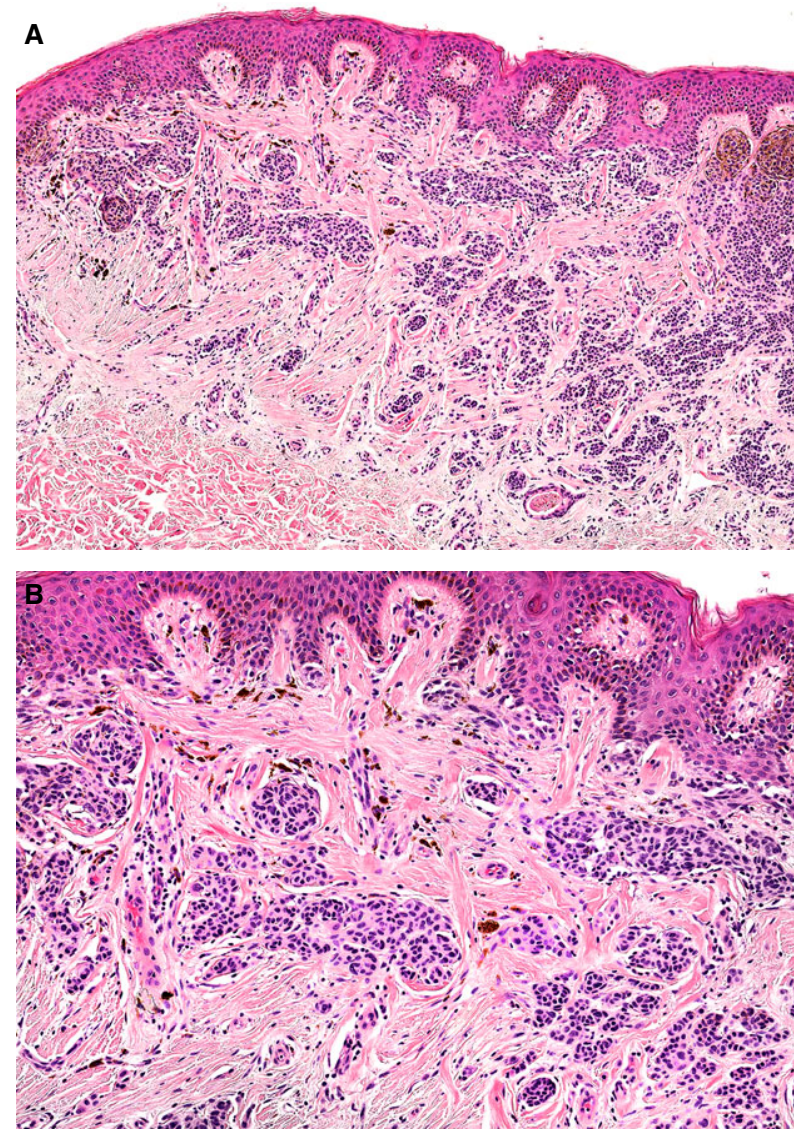

Figure 2. Atypical umbilical naevus (AUN). A, This compound naevus shows prominent lamellar fibrosis extending into the reticular dermis and entrapping most of the dermal nests in this field. B, Small junctional nests are confined mainly to the tips of the rete ridges. The dermal nests are slightly distorted and disposed haphazardly in the laminated collagenous stroma. Both the junctional and dermal melanocytes demonstrate minimal cytological atypia in this case.

AUN. Pagetoid scatter is a rare finding which, when present, should be focal and limited to the centre of the lesion. Cytologically, the junctional melanocytes typically demonstrate at least moderate atypia, with increase in cell size, 'dusty' cytoplasm, enlarged and hyperchromatic nuclei and variably prominent nucleoli.

Like other special site naevi, the atypical features in AUN may be concerning for melanoma. For example, pagetoid spread and involvement of the suprapapillary plates are present in a small subset of AUN; however, these findings are focal and limited. Junctional confluence and florid pagetoid spread should not be seen. Cases showing cytological atypia of the dermal component may also pose a significant diagnostic challenge. While the dermal cytological atypia is usually mild, some cases may exhibit a higher degree of atypia (Fig- 
A
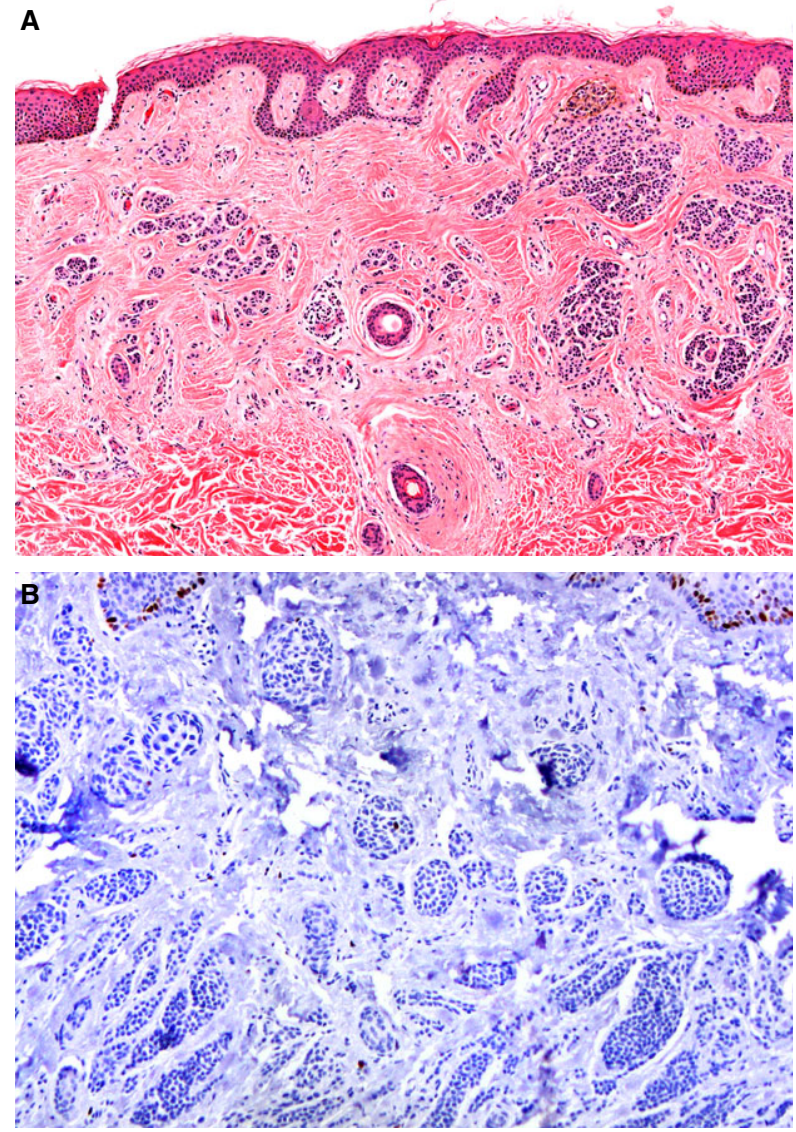

Figure 3. Atypical umbilical naevus (AUN). A, Broad and confluent lamellar fibrosis encompassing this entire intradermal naevus. B, Only rare dermal melanocytes were highlighted by Ki-67 immunostain, corresponding to a proliferation index of approximately $1 \%$. Nuclear staining of the basal keratinocytes serves as positive internal control.

ures 4 and 5). When evaluating AUN, it is important to ensure that the cytological atypia of the dermal component is restricted to the areas of lamellar fibrosis, and that the degree of atypia is not severe. Another reassuring finding is the lack of dermal mitoses in all AUN examined in this study. Although various studies have demonstrated dermal mitoses in benign naevi, ${ }^{18-}$ ${ }^{20}$ we suggest that one should remain cautious when faced with any atypical umbilical melanocytic lesion with dermal mitotic activity, especially when more than one mitosis is identified. Ancillary tools may be necessary in such challenging cases. We found a consistently low Ki-67 proliferation index of $<1 \%$ in the dermal component of 12 AUN, which provides additional evidence for benignity. ${ }^{21}$ Another commonly used immunostain is HMB45, which is expected to show a stratified staining pattern in benign naevi. ${ }^{22}$ More advanced molecular tools such as fluorescence in-situ hybridization have also proved helpful in the
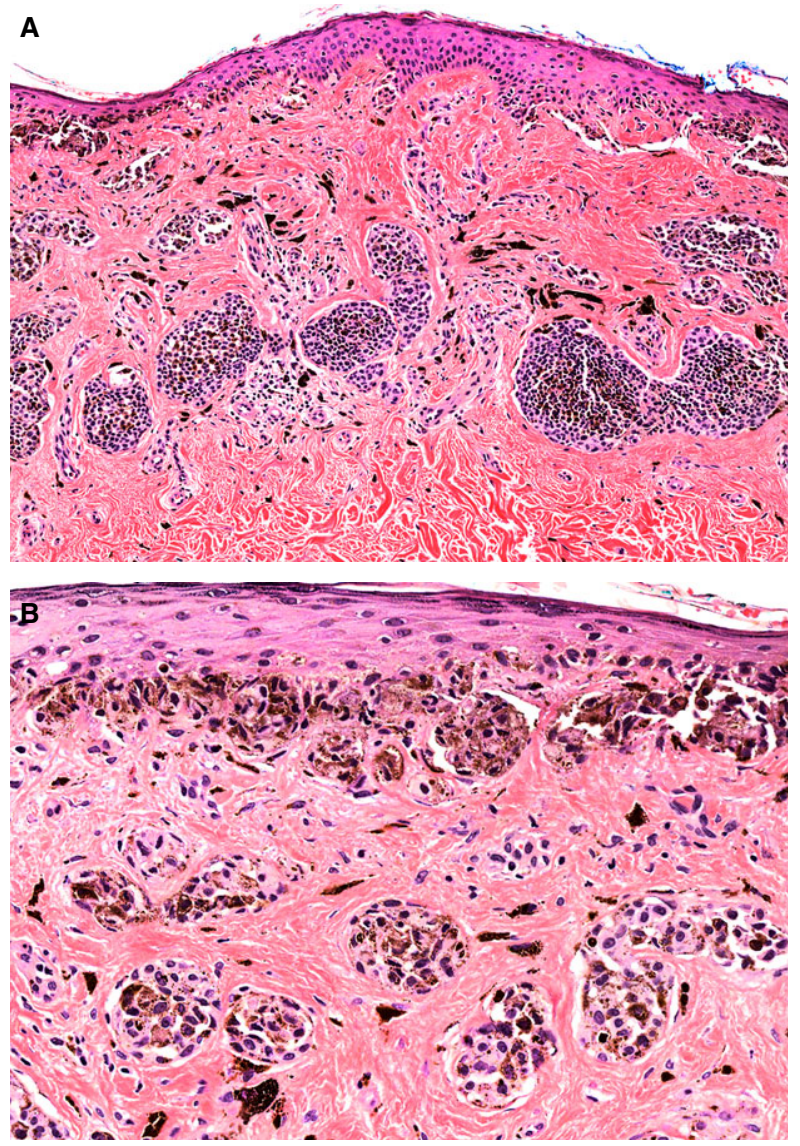

Figure 4. Atypical umbilical naevus (AUN). A, This lesion displays a more haphazard growth pattern. Dermal nests of variable sizes and shapes are associated with a fibrotic stroma. B, Focally, the junctional component shows nearly contiguous growth of lentiginous and nested melanocytes with high-grade atypia. The underlyling entrapped dermal nests exhibit a similar degree of atypia in this field.

differential diagnosis of naevi and melanoma. ${ }^{23,24}$ Lastly, careful examination for evidence of maturation outside the fibrotic areas would help greatly in distinguishing AUN from umbilical melanoma.

Similarities also exist between AUN and dysplastic naevi, including shoulder architecture and bridging. However, dysplastic naevi typically show more limited concentric or lamellar fibroplasia ${ }^{16,17}$ composed of only a few layers of delicate collagen fibres concentrically surrounding or lying flat below the tips of the rete ridges. These changes generally do not extend beyond the papillary dermis in dysplastic naevi, while the lamellar fibrosis in AUN is more confluent and often extends into the reticular dermis. Furthermore, lamellar fibrosis in AUN frequently entraps dermal nests, a feature typically absent in dysplastic naevi. As discussed before, these entrapped dermal nests may appear atypical and 
A
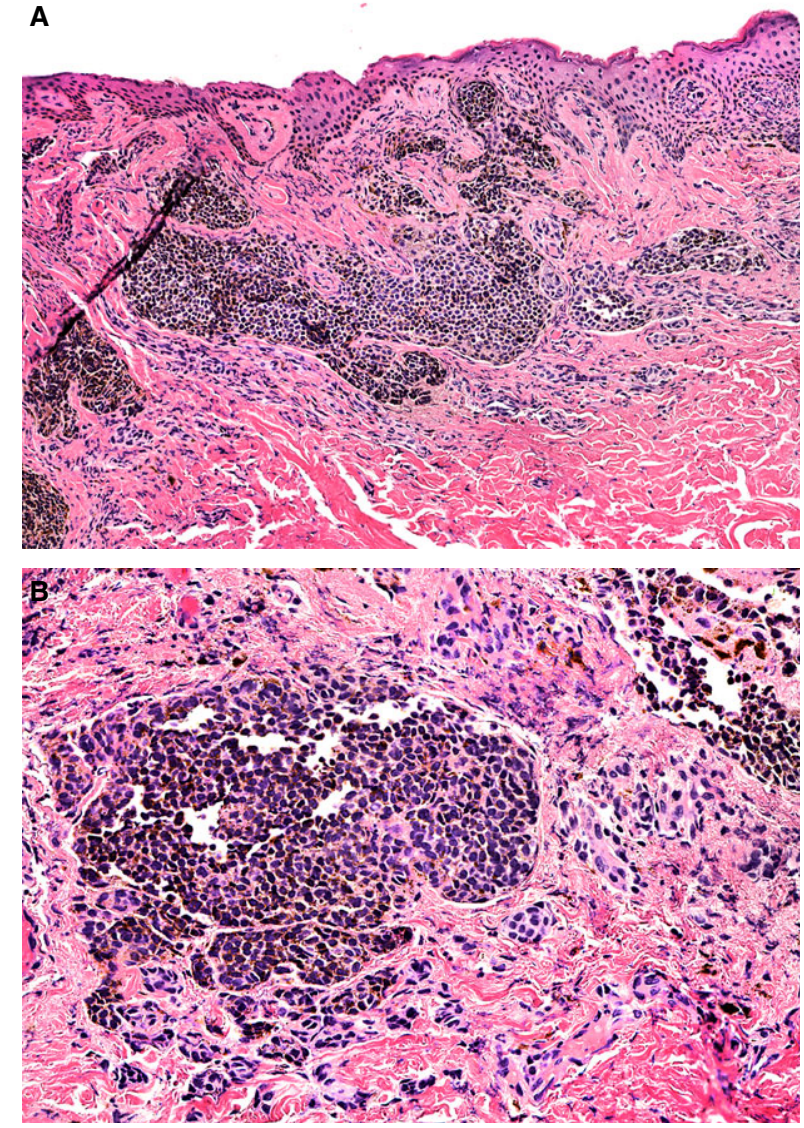

Figure 5. Atypical umbilical naevus (AUN). A, The dermal component of this compound naevus is markedly distorted by fibrosis. The entrapped dermal nests are large, confluent and irregular in shape. B, The melanocytes within a large dermal nest exhibit moderate cytological atypia with dusty cytoplasm and slightly hyperchromatic nuclei. Few melanocytes present beneath this large nest demonstrate some evidence of maturation (dispersion and diminution in cell size) outside the area of fibrosis.

may, at times, raise suspicion for partially regressed melanoma. In contrast, these findings are not characteristic of dysplastic naevi, as the lamellar fibroplasia rarely entraps melanocytes except in the variant showing 'florid fibroplasia associated with pseudomelanomatous features, ${ }^{25}$ which is discussed in detail below.

The finding of lamellar fibrosis with entrapped melanocytes in our series shares some similarities to the trizonal pattern described in 'sclerosing naevi with pseudomelanomatous features (SNPF)', which consists of (i) an atypical melanocytic proliferation at the dermo-epidermal junction, (ii) dermal lamellar fibrosis entrapping nests of melanocytes, and (iii) adjacent banal naevus uninvolved by fibrosis. ${ }^{26}$ A similar pattern has been reported in a series of dysplastic naevi which were referred to as 'Clark/dysplastic nevi with florid fibroplasia associated with pseudomelanomatous features'. ${ }^{25}$ The SNPF differ from our series, in that they showed a central whitish area clinically suggestive of regression, and were located most frequently on the back, lower extremities and abdomen (none described as umbilical). ${ }^{26}$ While the cause of the fibrotic changes in SNPF or Clark/dysplastic naevi with florid fibroplasia remains elusive, a number of possible aetiologies have been proposed, including minor unnoticed trauma, chronic friction, partial regression and prior folliculitis. ${ }^{25-27}$ In contrast, we believe the lamellar fibrosis in AUN is primarily a consequence of the unique developmental anatomy of the umbilicus. This probably accounts for the relatively high incidence $(25 \%)$ of AUN among all umbilical naevi examined in our study, while the incidence of 'pseudomelanomatous changes' among common or dysplastic naevi is presumably much lower based on our experience.

AUN are also distinct from recurrent naevi following incomplete excision or non-surgical trauma. ${ }^{28-32}$ Clinically, none of our cases had been biopsied previously. Unlike the scars in recurrent naevi, which are often devoid of melanocytes, the lamellar fibrosis in AUN typically entraps dermal melanocytes without evidence of angiogenesis or increased fibroblasts. Epidermal effacement, another characteristic of a scar, ${ }^{32}$ was lacking in all but one case of AUN; in the latter case this finding was possibly a result of prior nonsurgical trauma.

None of our AUN cases recurred or demonstrated any adverse outcome during an average follow-up period of 48.9 months, supporting a benign clinical course. Nevertheless, complete excision is recommended for lesions showing significant atypia and positive margins, in order to avoid subsequent diagnostic challenge associated with recurrent naevus phenomenon.

In conclusion, our study has shown that a subset of special site naevi in the umbilical region demonstrate characteristic lamellar fibrosis, architectural disorder and cytological atypia. Distinction of these AUN from melanoma may be challenging, but recognition of their site-related atypical features would avoid overdiagnosis of melanoma.

\section{Acknowledgements}

The authors would like to thank Tina Fields for her help with immunohistochemistry. This work was supported by internal funding provided by the Department of Pathology, University of Michigan. 


\section{Competing interests}

The authors declare no conflicts of interest.

\section{References}

1. Christensen WN, Friedman KJ, Woodruff JD, Hood AF. Histologic characteristics of vulvar nevocellular nevi. J. Cutan. Pathol. 1987; 14; 87-91.

2. Clark WH, Hood AF, Tucker MA, Jampel RM. Atypical melanocytic nevi of the genital type with a discussion of reciprocal parenchymal-stromal interactions in the biology of neoplasia. Hum. Pathol. 1998; 29; S1-S4.

3. Gleason BC, Hirsch MS, Nucci MR et al. Atypical genital nevi. A clinicopathologic analysis of 56 cases. Am. J. Surg. Pathol. 2008; 32; 51-57.

4. Fisher KR, Maize JC Jr, Maize JC Sr. Histologic features of scalp melanocytic nevi. J. Am. Acad. Dermatol. 2013; 68; 466-472.

5. Fabrizi G, Pagliarello C, Parente P, Massi G. Atypical nevi of the scalp in adolescents. J. Cutan. Pathol. 2007; 34; 365-369.

6. Lazova R, Lester B, Glusac EJ, Handerson T, McNiff J. The characteristic histopathologic features of nevi on and around the ear. J. Cutan. Pathol. 2005; 32; 40-44.

7. Saad AG, Patel S, Mutasim DF. Melanocytic nevi of the auricular region: histologic characteristics and diagnostic difficulties. Am. J. Dermatopathol. 2005; 27; 111-115.

8. Rongioletti F, Urso C, Batolo D et al. Melanocytic nevi of the breast: a histologic case-control study. J. Cutan. Pathol. 2004; 31; 137-140.

9. Boyd AS, Rapini RP. Acral melanocytic neoplasms: a histologic analysis of 158 lesions. J. Am. Acad. Dermatol. 1994; 31; 740-745.

10. Clemente C, Zurrida S, Bartoli C, Bono A, Collini P, Rilke F. Acral-lentiginous naevus of plantar skin. Histopathology 1995; 27; 549-555.

11. Rongioletti F, Ball RA, Marcus R, Barnhill RL. Histopathological features of flexural melanocytic nevi: a study of 40 cases. $J$. Cutan. Pathol. 2000; 27; 215-217.

12. Nicolau AA, Aşchie M. Morphologic and immunohistochemical features of breast nevi. Rom. J. Morphol. Embryol. 2013; 54; 371-375.

13. Weinstock MA, Barnhill RL, Rhodes AR, Brodsky GL. Reliability of the histopathologic diagnosis of melanocytic dysplasia. The Dysplastic Nevus Panel. Arch. Dermatol. 1997; 133; 953-958.

14. Mason AR, Mohr MR, Koch LH, Hood AF. Nevi of special sites. Clin. Lab. Med. 2011; 31; 229-242.

15. Conze J, Prescher A, Schlachter M, Schumacher O. The umbilical hernia. In Recurrent hernia: prevention and treatment, 1st edn. New York, NY: Springer, 2007; 359-364.

16. Clark WH Jr, Reimer RR, Greene M, Ainsworth AM, Mastrangelo MJ. Origin of familial malignant melanomas from heritable melanocytic lesions. 'The B-K mole syndrome'. Arch. Dermatol. 1978; 114; 732-738.

17. Elder DE. Dysplastic naevi: an update. Histopathology 2010; 56.112-120

18. Jensen SL, Radfar A, Bhawan J. Mitoses in conventional melanocytic nevi. J. Cutan. Pathol. 2007; 34; 713-715.

19. Nasr MR, El-Zammar O. Comparison of pHH3, Ki-67, and surviving immunoreactivity in benign and malignant melanocytic lesions. Am. J. Dermatopathol. 2008; 30; 117-122.

20. Ruhoy SM, Kolker SE, Murry TC. Mitotic activity within dermal melanocytes of benign melanocytic nevi: a study of 100 cases with clinical follow-up. Am. J. Dermatopathol. 2011; 33; 167-172.

21. Nasr MR, El-Zammar O. Comparison of pHH3, Ki-67, and survivin immunoreactivity in benign and malignant melanocytic lesions. Am. J. Dermatopathol. 2008; 31; 117-122.

22. Skelton HG III, Smith KJ, Barrett TL, Lupton GP, Graham JH. HMB-45 staining in benign and malignant melanocytic lesions. A reflection of cellular activation. Am. J. Dermatopathol. 1991; 13; 543-550.

23. Gerami P, Jewell SS, Morrison LE et al. Fluorescence in situ hybridization (FISH) as an ancillary diagnostic tool in the diagnosis of melanoma. Am. J. Surg. Pathol. 2009; 33; 1146-1156.

24. Gerami P, Wass A, Mafee M et al. Fluorescence in situ hybridization for distinguishing nevoid melanomas from mitotically active nevi. Am. J. Surg. Pathol. 2009; 33; 1783-1788.

25. Ko CJ, Bolognia JL, Glusac EJ. 'Clark/dysplastic' nevi with florid fibroplasia associated with pseudomelanomatous features. J. Am. Acad. Dermatol. 2011; 64; 346-351.

26. Fabrizi G, Pennacchia I, Pagliarello C, Massi G. Sclerosing nevus with pseudomelanomatous features. J. Cutan. Pathol. 2008; 35; 995-1002.

27. Ferrara G, Amantea A, Argenziano G et al. Sclerosing nevus with pseudomelanomatous features and regressing melanoma with nevoid features. J. Cutan. Pathol. 2009; 36; 913-915.

28. Kornberg R, Ackerman AB. Pseudomelanoma: recurrent melanocytic nevus following partial surgical removal. Arch. Dermatol. $1975 ; 111 ; 1588-1590$.

29. Park HK, Leonard DD, Arrington JH III, Lund HZ. Recurrent melanocytic nevi: clinical and histologic review of 175 cases. J. Am. Acad. Dermatol. 1987; 17; 285-292.

30. Sexton M, Sexton CW. Recurrent pigmented melanocytic nevus. A benign lesion, not to be mistaken for malignant melanoma. Arch. Pathol. Lab. Med. 1991; 115; 122-126.

31. Selim MA, Vollmer RT, Herman CM, Pham TT, Turner JW. Melanocytic nevi with nonsurgical trauma: a histopathologic study. Am. J. Dermatopathol. 2007; 29; 134-136.

32. King R, Hayzen BA, Page RN, Googe PB, Zeagler D, Mihn MC Jr. Recurrent nevus phenomenon: a clinicopathologic study of 357 cases and histologic comparison with melanoma with regression. Mod. Pathol. 2009; 22; 611-617. 